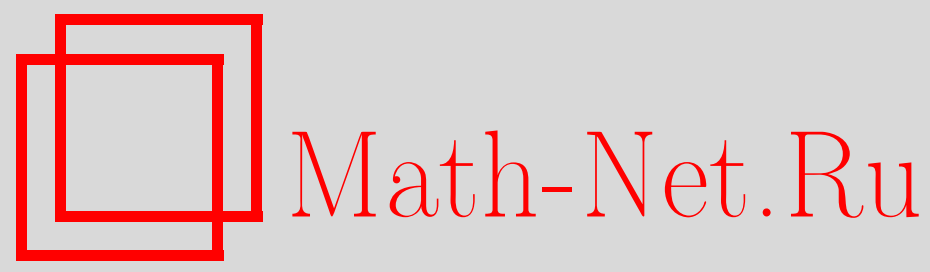

Р. Н. Мирошин, Асимптотическая оценка интеграла от произведения двух модифицированных бесселевых функций и степенной функции, Матем. заметки, 1997, том 61, выпуск $3,456-458$

DOI: https://doi.org/10.4213/mzm1522

Использование Общероссийского математического портала Math-Net.Ru подразумевает, что вы прочитали и согласны с пользовательским соглашением http://www . mathnet.ru/rus/agreement

Параметры загрузки:

IP : 3.95 .254 .165

26 апреля 2023 г., $12: 30: 48$

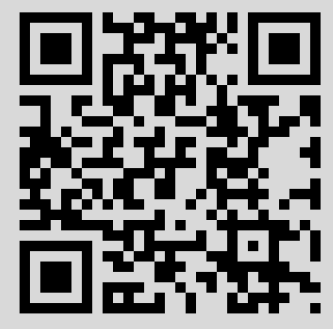




\section{АСИМПТОТИЧЕСКАЯ ОЦЕНКА ИНТЕГРАЛА ОТ ПРОИЗВЕДЕНИЯ ДВУХ МОДИФИЦИРОВАННЫХ БЕССЕЛЕВЫХ ФУНКЦИЙ И СТЕПЕННОЙ ФУНКЦИИ}

\section{Р.Н. Мирошин}

Найдем главньй член асимптотики при $\rho \uparrow 1$ интеграла

$$
I_{a}^{\rho}(\mu, \nu)=\int_{0}^{\infty} x^{-\rho} K_{i \mu}(x) K_{i \nu}(a x) d x,
$$

в котором $K_{i \mu}(x)$ - модифицированная функция Бесселя [1], $a>0, \mu>0, \nu>0$. C необходимостью вычисления (1) для $\rho \uparrow 1, a \neq 1$ мы встретились при упрощении континуального интеграла, представляющего функцию распределения числа нулей гауссовского марковского процесса первого порядка на конечном интервале времени [2], [3]. В случае $a=1$ асимптотика интеграла (1) при $\rho \uparrow 1$ известна давно (она лежит в основе преобразования Конторовича-Лебедева [4])

$$
I_{1}^{\rho}(\mu, \nu) \rightarrow \frac{\pi^{2}}{2 \mu \operatorname{sh} \pi \mu} \delta(\nu-\mu),
$$

где $\delta(x)$ - дельта-функция. В настоящей заметке мы выводим формулу, обобщающую (2) на случай произвольного $a>0$. Надеемся, что она (как и преобразование Конторовича-Лебедева) будет полезна не только в теории вероятностей.

В результат войдут обобщенные функции $\delta(x)$ и $\mathrm{Vp} \frac{1}{x}$, определяемые как линейные функционалы на пространстве $K$ бесконечно дифференцируемых функций $\varphi(x)$ с компактными носителями [5],

$$
\begin{aligned}
(\delta(x), \varphi(x)) & =\int_{R} \varphi(x) \delta(x) d x=\varphi(0), \quad \varphi(x) \in K, \\
\left(\mathrm{Vp} \frac{1}{x}, \varphi(x)\right) & =\int_{R} \varphi(x) \mathrm{Vp} \frac{1}{x} d x=\mathrm{Vp} \int_{R} \frac{\varphi(x)}{x} d x, \quad \varphi(x) \in K,
\end{aligned}
$$

где $R=(-\infty, \infty)$, а символом $\mathrm{Vp}$ перед интегралом обозначается главное его значение в смысле Коши

$$
\mathrm{Vp} \int_{\alpha}^{\beta} \frac{\varphi(x)}{x} d x=\lim _{\varepsilon \downarrow 0}\left(\int_{-\alpha}^{-\varepsilon} \frac{\varphi(x)}{x} d x+\int_{\varepsilon}^{\beta} \frac{\varphi(x)}{x} d x\right), \quad \alpha>0, \quad \beta>0 .
$$

Теорема. При $\rho \uparrow 1, a>0, \mu>0, \nu>0$ справедливо соотношение

$$
\begin{gathered}
I_{a}^{\rho}(\mu, \nu) \rightarrow \frac{\pi}{(\mu+\nu) \operatorname{sh}(\pi(\mu+\nu) / 2)}\left[\pi \delta(\nu-\mu) \cos \left(\frac{\mu+\nu}{2} \ln a\right)\right. \\
\left.+\sin \left(\frac{\mu+\nu}{2} \ln a\right) \operatorname{Vp} \frac{1}{\nu-\mu}+f_{a}(\mu, \nu)\right],
\end{gathered}
$$

в котором

$$
f_{a}(\mu, \nu)=\int_{0}^{1} \sin \left[\frac{\nu-\mu}{2} \ln x+\frac{\nu+\mu}{4} \ln \frac{a(x+a)}{1+a x}\right] \sin \left[\frac{\nu+\mu}{4} \ln \frac{a(1+a x)}{x+a}\right] \frac{d x}{x},
$$

а предел понимается как

$$
\lim _{\rho \uparrow 1}\left(I_{a}^{\rho}(\mu, \nu), \varphi(\nu)\right), \quad \varphi(\nu) \in K .
$$

Очевидно, формула (2) - частньй случай (3) при $a=1$. 
ДоКАЗАТЕЛЬСТВо. Согласно [1, с. 736, № 6648]

$$
K_{i \mu}(x) K_{i \nu}(a x)=\int_{-\infty}^{\infty} e^{i(\nu-\mu) t}\left(\frac{e^{t}+a e^{-t}}{e^{-t}+a e^{t}}\right)^{i(\mu+\nu) / 2} K_{i(\mu+\nu)}(x b) d t
$$

где $b=\sqrt{1+2 a \operatorname{ch} 2 t+a^{2}}$. Подставив (4) в $(1)$, обозначив $\alpha=(1-\rho) / 2, \beta=(\nu-\mu) / 2$, $\gamma=(\nu+\mu) / 2$ и поменяв местами порядок интегрирования по $x$ и $t$, имеем

$$
I_{a}^{\rho}(\mu, \nu)=\int_{-\infty}^{\infty} d t e^{2 i t \beta}\left(\frac{e^{t}+a e^{-t}}{e^{-t}+a e^{t}}\right)^{i \gamma} \int_{0}^{\infty} d x x^{-\rho} K_{i 2 \gamma}(x b) .
$$

Внутренний интеграл в (5) - табличный $[1$, с. 698 , № 6561.16]

$$
\int_{0}^{\infty} x^{-\rho} K_{i 2 \gamma}(x b) d x=4^{\alpha-1} b^{-2 \alpha} \Gamma(\alpha+i \gamma) \Gamma(\alpha-i \gamma)
$$

и поэтому из (5) следует

$$
\begin{gathered}
I_{a}^{\rho}(\mu, \nu)=4^{\alpha-1} \Gamma(\alpha+i \gamma) \Gamma(\alpha-i \gamma) F_{a}^{\alpha}(\beta, \gamma) \\
F_{a}^{\alpha}(\beta, \gamma)=\int_{-\infty}^{\infty} \frac{e^{2 i t \beta} \psi_{a}^{\gamma}\left(e^{t}\right) d t}{\left(1+2 a \operatorname{ch} 2 t+a^{2}\right)^{\alpha}}, \quad \psi_{a}^{\gamma}(t)=\left(\frac{x^{2}+a}{1+a x^{2}}\right)^{i \gamma}
\end{gathered}
$$

При $\alpha \downarrow 0$ знаменатель в (7) ведет себя как $a^{\alpha} e^{2 \alpha|t|}(1+o(1))$, так что при $\alpha \downarrow 0$

$$
\begin{aligned}
F_{a}^{\alpha}(\beta, \gamma) & \sim \int_{-\infty}^{\infty} \exp (2 i t \beta-2|t| \alpha) \psi_{a}^{\gamma}\left(e^{t}\right) d t \\
& =\int_{0}^{\infty} e^{-2 t \alpha}\left[e^{2 i t \beta} \psi_{a}^{\gamma}\left(e^{t}\right)+\frac{e^{-2 i t \beta}}{\psi_{a}^{\gamma}\left(e^{t}\right)}\right] d t
\end{aligned}
$$

Заменим в (8) переменную интегрирования по формуле $e^{-2 t}=x$. Получим

$$
2 F_{a}^{\alpha}(\beta, \gamma) \sim \int_{0}^{1} x^{\alpha-1}\left\{x^{-i \beta}\left(\frac{1+a x}{x+a}\right)^{i \gamma}+x^{i \beta}\left(\frac{1+a x}{x+a}\right)^{-i \gamma}\right\} d x
$$

Подьнтегральная функция в (9) имеет особенность при $x=0$. Регуляризуем эту функцию в точке $x=0$, прибавив к фигурной скобке и вычтя из нее ее значение при $x=0$,

$$
\begin{aligned}
2 F_{a}^{\alpha}(\beta, \gamma) & \sim \int_{0}^{1} x^{\alpha-1}\left(x^{-i \beta} a^{-i \gamma}+x^{i \beta} a^{i \gamma}\right) d x+\varphi_{a}^{\alpha}(\beta, \gamma) \\
& =\frac{a^{-i \gamma}}{\alpha-i \beta}+\frac{a^{i \gamma}}{\alpha+i \beta}+\varphi_{a}^{\alpha}(\beta, \gamma),
\end{aligned}
$$

где

$$
\varphi_{a}^{\alpha}(\beta, \gamma)=\int_{0}^{1} x^{\alpha}\left\{x^{-i \beta-1}\left[\left(\frac{1+a x}{x+a}\right)^{i \gamma}-a^{-i \gamma}\right]+x^{i \beta-1}\left[\left(\frac{1+a x}{x+a}\right)^{-i \gamma}-a^{i \gamma}\right]\right\} d x
$$


Переходя к пределу $\alpha \downarrow 0$ в (11), получаем из $(10),(11)$

$$
\begin{gathered}
2 F_{a}^{\alpha}(\beta, \gamma) \sim \frac{2 \alpha \cos (\gamma \ln a)}{\alpha^{2}+\beta^{2}}+\frac{2 \beta \sin (\gamma \ln a)}{\alpha^{2}+\beta^{2}}+\varphi_{a}^{0}(\beta, \gamma), \\
\varphi_{a}^{0}(\beta, \gamma)=2 \int_{0}^{1}\left[\cos \left(\beta \ln x-\gamma \ln \frac{1+a x}{x+a}\right)-\cos (\beta \ln x+\gamma \ln a)\right] \frac{d x}{x} \\
=4 \int_{0}^{1} \sin \left[\beta \ln x+\frac{\gamma}{2} \ln \frac{a(x+a)}{1+a x}\right] \sin \left[\frac{\gamma}{2} \ln \frac{a(1+a x)}{x+a}\right] \frac{d x}{x}
\end{gathered}
$$

В окрестности точки $x=0$ имеем в $(13)$

$$
\frac{a(1+a x)}{x+a} \sim 1+\frac{a^{2}-1}{a} x, \quad \ln \frac{a(1+a x)}{x+a} \sim x \frac{\left|a^{2}-1\right|}{a} \operatorname{sign}\left(a^{2}-1\right) .
$$

Поэтому для $x \in[0,1]$

$$
\left|\frac{1}{x} \sin \left[\frac{\gamma}{2} \ln \frac{a(1+a x)}{x+a}\right]\right| \leqslant \text { const }<\infty,
$$

так что интеграл (13) конечен.

Вернемся к (12). Известно [3, с. 52, 414], что при $\alpha \downarrow 0$

$$
\frac{\alpha}{\alpha^{2}+\beta^{2}} \rightarrow \pi \delta(\beta), \quad \frac{1}{i \alpha \pm \beta} \rightarrow \pm \mathrm{Vp} \frac{1}{\beta}-i \pi \delta(x) .
$$

Поэтому

$$
\frac{\beta}{\alpha^{2}+\beta^{2}}=\frac{1}{2}\left(\frac{1}{\beta+i \alpha}+\frac{1}{\beta-i \alpha}\right) \rightarrow \mathrm{Vp} \frac{1}{\beta},
$$

и мы получаем из (12) при $\alpha \downarrow 0$

$$
F_{a}^{\alpha}(\beta, \gamma) \rightarrow \pi \delta(\beta) \cos (\gamma \ln a)+\sin (\gamma \ln a) \operatorname{Vp} \frac{1}{\beta}+\frac{1}{2} \varphi_{a}^{0}(\beta, \gamma)
$$

Так как при $\alpha \downarrow 0[4$, с. 17$]$

$$
\Gamma(\alpha-i \gamma) \Gamma(\alpha+i \gamma) \rightarrow \Gamma(-i \gamma) \Gamma(i \gamma)=\frac{\pi}{\gamma \operatorname{sh} \pi \gamma}
$$

то, подставляя (14), (15) в (6) и раскрьвая $\beta$ и $\gamma$ через $\mu$ и $\nu$, приходим к утверждению теоремы.

Санкт-Петербургский государственный университет

Поступило 12.09 .96

\section{СПИСОК ЦИТИРОВАННОЙ ЛИТЕРАТУРЫ}

1. Градштейн И. С., Рыжик И. М. Таблицы интегралов, сумм, рядов и произведений. М.: Наука, 1971. 2. Мирошин Р. Н. // Теория вероятн. и ее примен. 1981. Т. 26. №1. С. 101-120. 3. Мирошин Р. Н. Пересечения кривых гауссовскими процессами. Л.: Изд-во ЛГУ, 1981. 4. Бейтмен Г., Эрдейи А. Высшие трансцендентные функции. Т. 1. М.: Наука, 1965. 5. Кеч В., Теодореску П. Введение в теорию обобщенных функций с приложениями в технике. М.: Мир, 1978. 6. Гельфанд И. М., Шилов Г. Е. Обобщенные функции и действия над ними. Вьп. 1. М.: Физматгиз, 1959. 